\title{
CORRIGENDUM
}

\section{Nasal intermittent positive pressure ventilation in the newborn: review of literature and evidence-based guidelines}

\section{Bhandari}

Journal of Perinatology (2010) 30, 827; doi:10.1038/jp.2010.117

Correction to: Journal of Perinatology (2009) 30, 505-512; doi:10.1038/jp.2009.165; published online 22 October 2009
The article stated that SNIPPV was significantly better than NCPAP in preventing extubation failure within $72 \mathrm{~h}$ (and even after including 'late failures') in 154 enrolled infants who recovered from RDS. The correct number of infants enrolled was 64. 